\title{
MODÉLISATION DE LA MORPHODYNAMIQUE FLUVIALE POUR LA RECHERCHE DES RELATIONS HABITAT/FAUNE AQUATIQUE.
}

\author{
Y. LE COARER et B. DUMONT \\ CEMAGREF, Division Hydrobiologie, Le Tholonet, BP 31, \\ 13612 Aix-en-Provence Cedex 1, France.
}

\section{RÉSUMÉ}

Un matériel de topographie électronique et des méthodes adaptées nous procurent rapidité, précision et souplesse dans le repérage spatial des éléments morphodynamiques et biologiques. Le traitement informatique des données fait appel à des techniques de repérage curviligne d'interpolation et de maillage. La structure numérique et topologique de cet outil de recherche offre de nombreuses possibilités de calculs et permet des visualisations conviviales des résultats.

\section{MODELLING STREAM MORPHODYNAMICS FOR RESEARCH IN HABITAT/AQUATIC FAUNA RELATIONSHIPS.}

\section{SUMMARY}

An electronic topography equipment and adapted methods provided rapidity, accuracy and flexibility in the spatial location of hydrodynamic and biological data. Data processing used curvilinear location techniques of interpolation and gridding. The numerical and topological structure of this research tool offers numerous calculation possibilities and insures convivial presentations of the results.

\section{INTRODUCTION}

Afin de prévoir les effets des aménagements sur la vie des cours d'eau, nous cherchons à modéliser ces hydrosystèmes et en particulier les relations habitats/poissons. L'outil présenté ici permet une quantification des facteurs d'habitat qui régissent les populations aquatiques. Les différentes échelles de perception spatiale pouvant être utilisées, s'emboîtent du mètre au kilomètre. Elles s'étagent de l'habitat du benthos au tronçon de rivière.

Nous avons, tout d'abord, adapté des techniques de topographie électronique à la description morphodynamique de rivière et à la localisation de données biologiques. Puis, nous avons réalisé un travail informatique qui s'apparente beaucoup à la constitution d'un Système d'Information Géographique. Le Modèle Numérique de Terrain (MNT) employé pour décomposer les cours d'eau est constitué d'un maillage en prismes droits à bases triangulaires. Cette démarche MNT permet, entre autres, des calculs hydrauliques de type éléments finis ; elle a aussi été appliquée pour la modélisation de l'habitat piscicole par LECLERC et al. (1994).

L'originalité de notre travail réside dans la méthode de repérage curviligne, les techniques d'interpolation et dans la génération de profils en travers réputés perpendiculaires à l'écoulement. Les possibilités topologiques qui gèrent indifféremment mailles, transversales et polygones permettent de s'extraire de tout "a priori" de structures et de rechercher les corrélations et les statistiques qui représentent le mieux le phénomène observé. 


\section{MATÉRIEL ET MÉTHODES DE TERRAIN}

La base de la méthode consiste à repérer spatialement les éléments descriptifs de la rivière, qu'ils soient de nature morphodynamique ou biologique.

Le matériel utilisé comprend :

- un tachéomètre associant un théodolite électronique Théomat WILD-LEITZ T1000 à un distancemètre WILD DI 1000 à onde porteuse infrarouge ; l'ensemble est relié à un carnet électronique WILD GRE3,

- un prisme réflecteur auto-convergent supporté par une canne,

- une liaison radio entre les opérateurs décrivant le cours d'eau et la station topographique où est implanté le tachéomètre.

Depuis le tachéomètre, on vise le prisme définissant un point dont la position relative est définie par une distance oblique, un angle horizontal, un angle vertical, les hauteurs du prisme et de l'appareil par rapport au sol. Le carnet électronique gère en mémoire vive un code d'identification et un numéro de point à incrément automatique. Ces informations, modifiables à volonté, permettent le repérage (rive droite, berge droite, etc.) et la description séquentielle indexée des unités topologiques telles que les rives et les polygones décrivant les îles, les zones d'isogranulométrie et d'échantillonnage (poissons, benthos). Après déclenchement de la visée, l'enregistrement des coordonnées sphériques du point et des informations de la mémoire vive est automatique. En notant le numéro de point on peut référencer dans l'espace toutes les mesures locales, comme la profondeur ou la vitesse de courant. Le carnet électronique offre la possibilité d'enregistrement manuel de ces mesures.

Pour la topographie de la morphologie de la rivière nous utilisons une technique de semis densifié ; la densité de points relevés est ainsi fonction de la complexité de la description.

Les points rivulaires à l'interface eau/berge définissent la surface de l'eau ; aussi, hormis dans les écoulements torrentiels, il n'est pas nécessaire de noter les profondeurs qui peuvent être retrouvées par différence d'altitude entre le fond et la surface.

La rapidité de description est variable car fonction de la densité végétale de la ripisylve et de la pénétrabilité du cours d'eau. Pour des profondeurs supérieures à $1.20 \mathrm{~m}$, l'utilisation d'un bateau et de prismes flottants associée à des mesures de profondeur s'avère nécessaire. Cette technique apparaît 2 à 3 fois plus rapide que les systèmes classiques avec niveau optique et mires.

La description du lit mouillé doit nécessairement être associée à la connaissance du débit. Dans des situations d'écoulement complexe, il est souhaitable de jauger la répartition des débits de part et d'autre des îles, car elle est souvent difficilement calculable par les modèles hydrauliques. Le suivi des variations de niveau d'eau pendant les opérations topographiques peut s'effectuer à l'aide d'une sonde de pression dont les mesures sont stockées dans une centrale d'acquisition. Pour minimiser les incertitudes liées à ces variations, la surface de l'eau devra être décrite en une phase et le plus rapidement possible.

\section{LE TRAITEMENT INFORMATIQUE}

Les points suivants développent les caractéristiques essentielles des procédures, que nous avons toutes développées et écrites en langage MICROSOFT QUICK BASIC 4.5.

\section{- Repérage curviligne}

Chaque verticale relevée sur le terrain est repérée dans l'espace, après traitement informatique, par:

- ses coordonnées cartésiennes $\mathbf{x}, \mathbf{y}, \mathbf{z}$ (fond),

- sa profondeur,

- ses coordonnées curvilignes $\mathbf{s}, \mathbf{b}$, be (Fig. 1 et les explications qui suivent). 


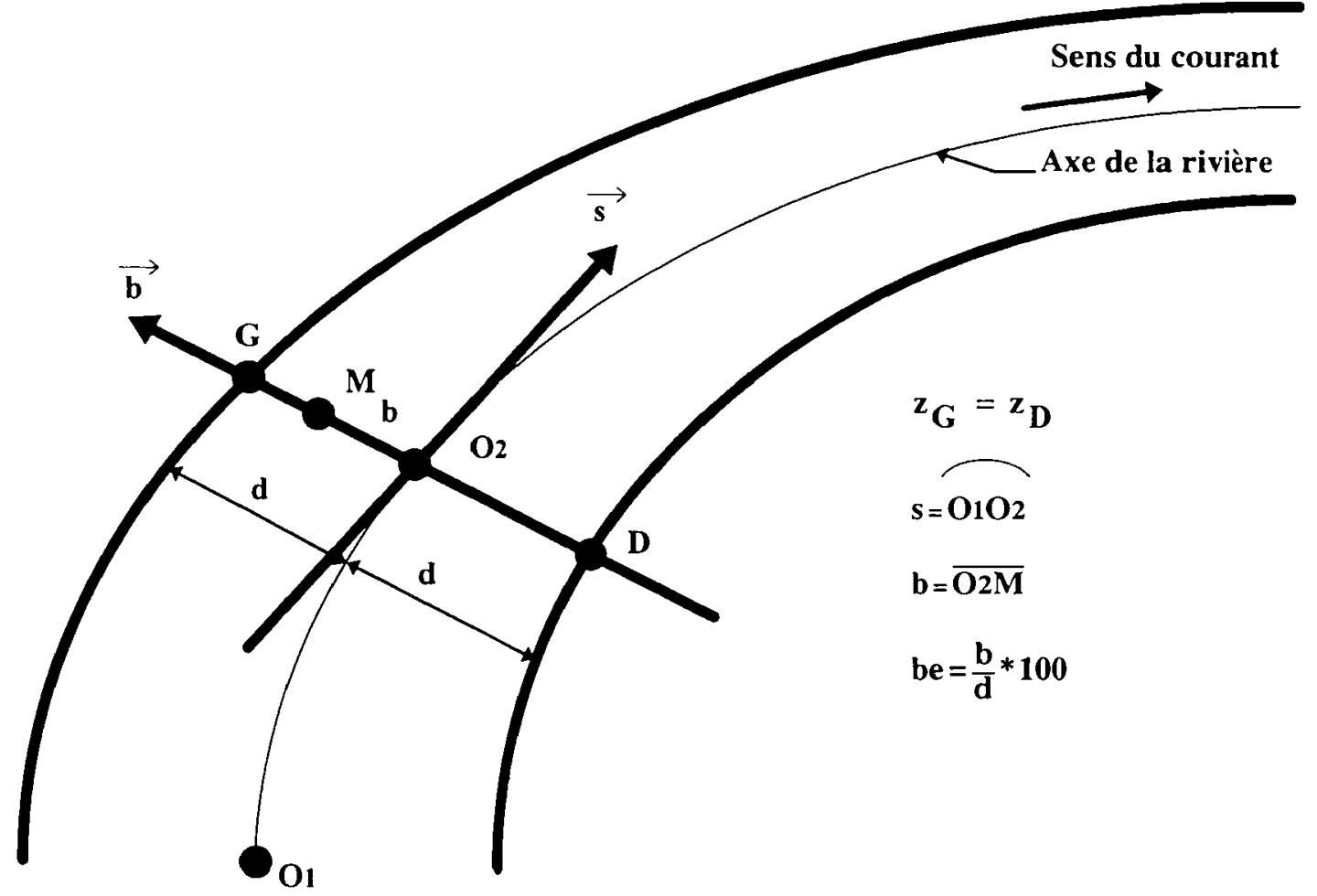

Figure 1 : Principe du repérage curviligne (cf. explications dans le texte).

Figure 1 : Principle of curvilinear location (see explanations in the text).

O1 : origine du système de balayage en abscisse curviligne.

$\mathrm{O} 2$ : origine locale du repère curviligne située au milieu du chenal. La détermination de l'axe de la rivière est une phase complexe, elle s'effectue dans le plan horizontal à partir des tracés des rives, tracés qu'il est souvent nécessaire de lisser.

$(\mathrm{O} 2,-\overrightarrow{\mathrm{O} 2 \mathrm{~s}}, \overrightarrow{\mathrm{O} 2 b})$ : repère normé curviligne tel que $\overrightarrow{\mathrm{O} 2 \mathrm{~s}}$ ne soit pas nécessairement perpendiculaire à $\stackrel{\mathbf{O} 2 \mathrm{~b}}{\longrightarrow}$ mais tangent à l'axe de la rivière. La détermination de

$\mathbf{O} 2 \mathbf{b}$ s'efforce de réaliser l'isoaltitude des deux points rivulaires ayant même abscisse curviligne $\left(\mathbf{z}_{\mathbf{G}}=\mathbf{z}_{\mathbf{D}}\right)$. Dans les mouilles où la pente de la surface de l'eau est difficile à estimer, $\overrightarrow{\mathbf{O 2 b}}$ est fixé par des considérations géométriques à partir du tracé dans le plan horizontal des rives.

Pour le point $M$, on définit :

- son abscisse curviligne s qui est la distance curviligne mesurée le long de l'axe de la rivière :

$$
s=\overparen{0102}
$$


- son ordonnée curviligne $\mathbf{b}$ comptée positivement depuis l'axe de la rivière vers la rive gauche:

$$
\mathbf{b}=\overline{\mathbf{O} 2 \mathrm{M}}
$$

- son pourcentage algébrique d'éloignement à l'axe de la rivière be, un point en rive gauche vérifie be $=100 \%$. un point en rive droite be $=-100 \%$ :

$$
\text { be }=\frac{b}{d} * 100
$$

où d est la demi-largeur de la riviere au niveau du transect passant par $\mathbf{0 2}$.

On dispose ainsi d'un procédé qui permet un classement amont aval des informations et la définition de plans réputés perpendiculaires à l'écoulement.

\section{- Interpolation}

Dans le domaine topographié et en toute position $(x, y)$, une seconde génération de points peut être calculée par interpolation. La méthode utilisée met en oeuvre des règles d'expertise issues de la méthodologie descriptive de terrain.

Dans un premier temps, on détermine leur profondeur. L'un des principes utilisés dans ces calculs, consiste à pondérer les profondeurs des points topographiés d'autant plus fortement que leur pourcentage d'éloignement à l'axe (be) est proche de celui du point interpolé. Ainsi, un point généré en bordure sera peu influencé par la profondeur d'un point situé au milieu du chenal, même si celui-ci est son plus proche voisin.

Les altitudes $\mathbf{z}$ (fond) des points interpolés sont ensuite obtenues par différence entre l'altitude de la surface de l'eau et la profondeur. Dans les secteurs à écoulements torrentiels, la surface de l'eau est définie à partir de points encadrant le point interpolé, ailleurs par le balayage curviligne isoaltitude s'appuyant sur les points rivulaires.

\section{- Profils en long et en travers}

La procédure d'interpolation permet la génération de profils en travers à la demande ; la précision de leur surface mouillée est d'environ $10 \%$. Les profils en travers fournissent la profondeur moyenne, la vitesse moyenne et l'altitude de la surface de l'eau le long de l'axe de la rivière. Ceci permet la détermination de la pente de la ligne d'énergie pour toute abscisse curviligne et l'établissement de profils en long.

\section{- Reconstitution de vitesses}

Dans les cours d'eau à faible profondeur et à forte rugosité relative, aux écoulements complexes (radier déversant, etc.), il est pratiquement impossible de calculer précisément des vitesses locales. En effet, réaliser cette opération nécessiterait l'emploi des codes de calcul les plus performants et une topographie trop détaillée où chaque bloc serait décrit. Si ces deux conditions étaient remplies, les temps de calculs pour $1 \mathrm{~km}$ de rivière seraient considérables, même sur les matériels les plus modernes. Face à ce problème, nous avons adopté une modélisation grossière des champs de vitesse associée à la caractérisation des rapports rugosité/profondeur. Pris ensemble, ces paramètres traduisent la diversité des vitesses et la présence de zones d'ombre hydraulique dans l'habitat aquatique.

Une vitesse moyenne est calculée pour tout point topographié et interpolé. Les principes de base de ce calcul consistent :

- à faire passer par chaque verticale un profil en travers,

- à calculer, pour chaque verticale du profil en travers, une vitesse moyenne en utilisant majoritairement la formule de LIMERINOS (1970), plus adaptée que celle de MANNINGSTRICKLER pour les rivières à faible profondeur,

- à réaliser un ajustement des vitesses moyennes pour retrouver le débit de la rivière dans le profil en travers. 
Afin d'effectuer ce type de calcul, il est préconisé d'avoir réalisé un découpage de la rivière en zones d'isogranulométrie, dans lesquelles ont été mesurées des rugosités. Ceci permet alors d'attribuer à chaque verticale située dans la zone :

- le k84 employé dans la formule de LIMERINOS : rugosité $(\mathrm{m})$ telle que $84 \%$ des éléments granulométriques ont une dimension inférieure à cette valeur.

- le rapport rugosité moyenne/hauteur d'eau moyenne.

\section{- Maillage}

Le maillage de la rivière, de type irrégulier, est réalisé en prismes droits à bases triangulaires (Fig. 3). Chaque prisme est composé de 3 verticales définissant un élément de volume dont les facettes supérieure et inférieure représentent respectivement la surface de l'eau et le fond. On affecte à chacun des prismes les valeurs moyennes des paramètres physiques des verticales. Le calcul des valeurs moyennes par zone s'effectue par agglomération de mailles élémentaires. Il utilise une pondération par volume dans le cas des vitesses moyennes par exemple, ou par la surface du fond dans le cas des contraintes tangentielles.

\section{- Exemple de descripteurs physiques locaux et d'ambiance}

Nous donnons ici un exemple des possibilités offertes, au travers d'une liste des variables utilisées pour rechercher des relations habitats/poissons (PARUSSIE, 1994 ; CARREL et al., 1994).

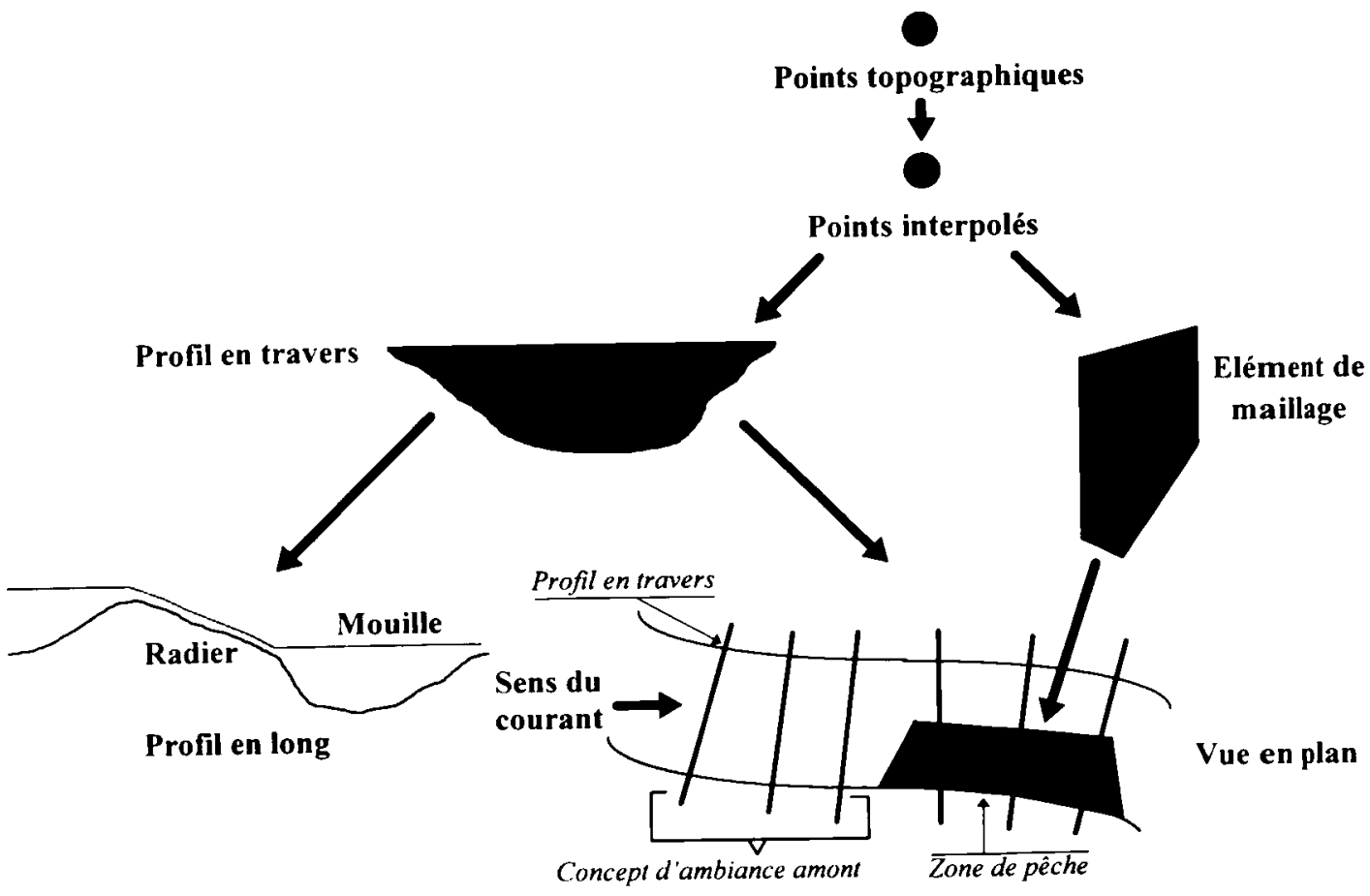

Figure 2 : Schéma de principe du traitement informatique des données.

Figure 2 : Principle diagram of data processing. 
Tableau I : Variables de description locale de la zone de pêche.

Table I : Variables for local description of the fishing zone.

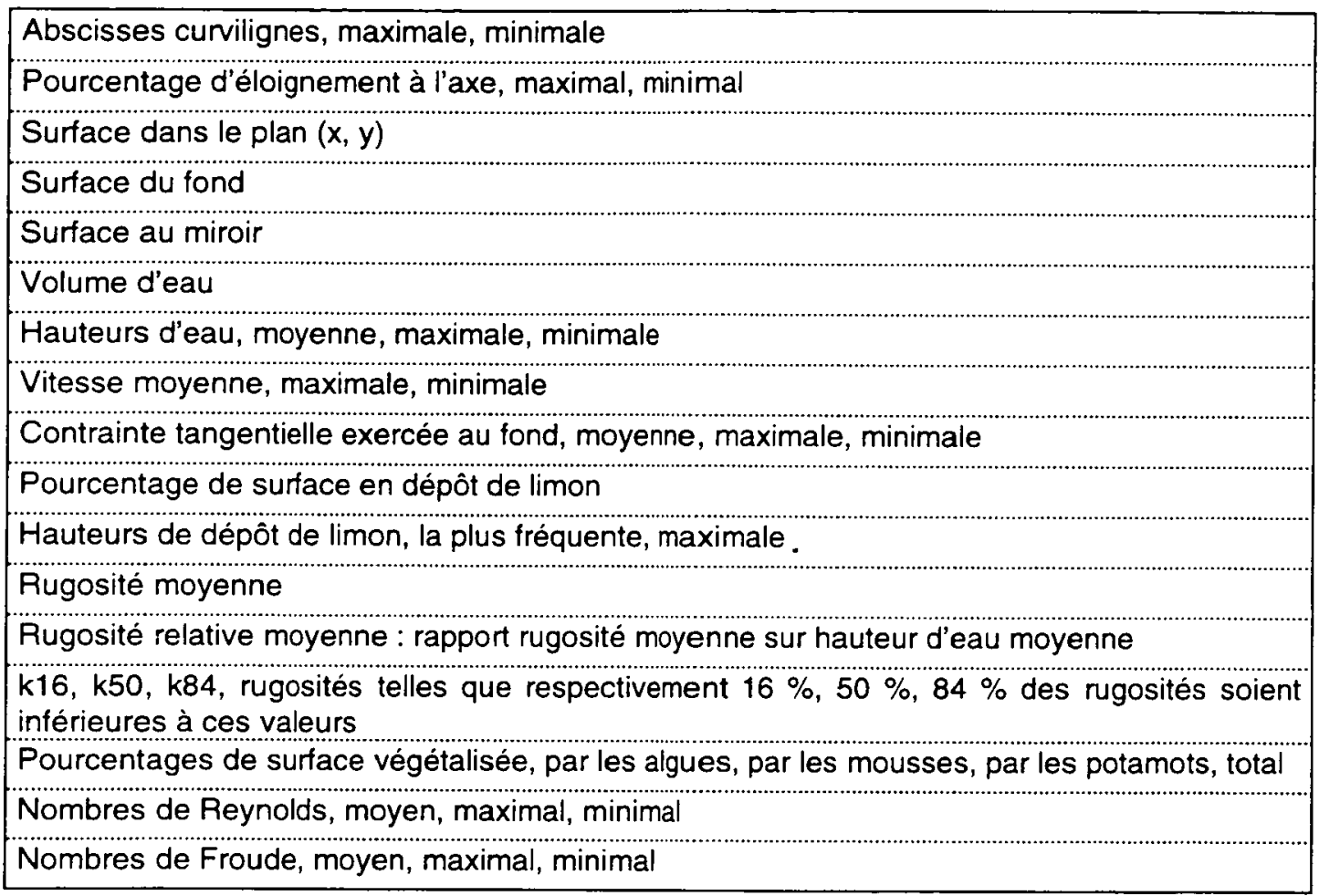

Tableau II : Variables de description de l'ambiance de la zone de pêche.

Table II : Surrounding habitat variables of the fishing zone.

\begin{tabular}{l} 
Moyennes issues des profils en travers coupant la zone de pêche \\
\hline largeurs au miroir \\
surfaces mouilées \\
périmètres mouillés \\
\hline hauteurs d'eau moyennes \\
\hline rayons hydrauliques \\
rapports largeur au miroir sur profondeur \\
\hline vitesses moyennes \\
\hline nombres de Froude moyens \\
\hline nombres de Reynolds moyens \\
\hline pentes de la surface de l'eau \\
pentes de la ligne d'énergie \\
\hline Moyennes issues des profils en travers répartis sur 10 mètres à l'amont de la zone de pêche \\
\hline nombres de Froude moyens \\
pentes de la surface de l'eau \\
rugosités relatives : rapports rugosités sur hauteurs d'eau \\
\hline
\end{tabular}




\section{- Représentations graphiques}

Des programmes informatiques conviviaux permettent :

— la visualisation des profils en travers le long de l'abscisse curviligne ; l'utilisateur peut les faire défiler à volonté et obtenir leurs caractéristiques,

- la visualisation de la rivière en plan, ou en profil en long ; l'utilisateur peut se déplacer et zoomer à volonté dans ces représentations. II peut choisir des palettes de couleur pour différencier les valeurs des variables morphodynamiques (Fig. 3). Des connaissances de base en programmation lui offrent la possibilité d'exprimer des requêtes telles que la coloration de zones selon un filtre de critères.

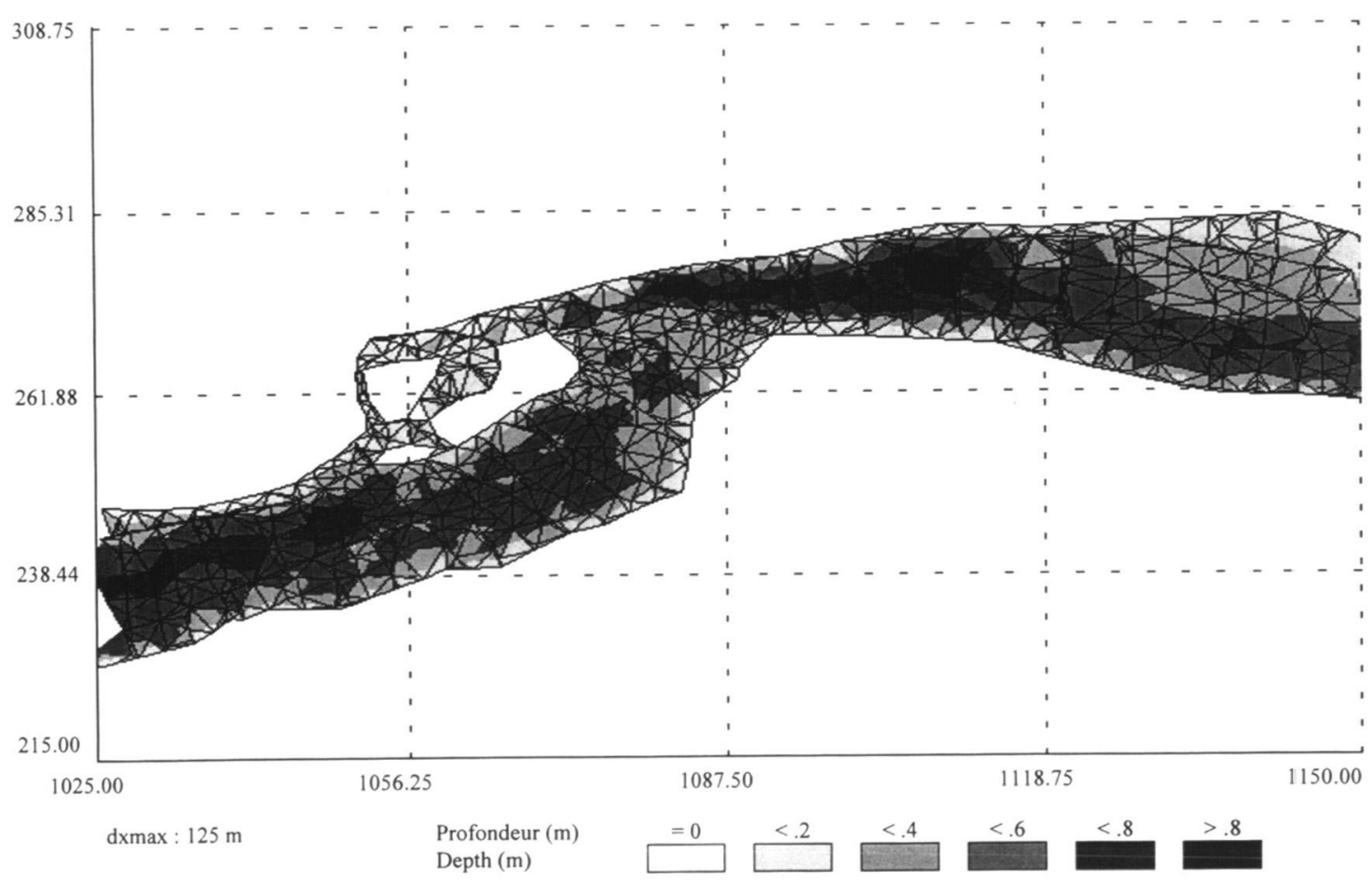

Figure 3 : Représentation $x-y(m)$ du maillage, visualisation des profondeurs.

Figure $3: x-y(m)$ display of grid and depth colouring.

\section{PERSPECTIVES}

Les procédures de repérage curviligne et de maillage, la structure des fichiers qui conditionne la maîtrise topologique des rives et des polygones (zones), la souplesse des repérages possibles sur le terrain, permettent à cet outil d'être très adaptable.

Nous avons pu ainsi, en quelques jours, ajouter un module de calcul et de visualisation des valeurs d'habitats pour 4 taxons de truite selon la méthode des microhabitats (BOVEE, 1981 et 1982 ; SOUCHON et al., 1989).

La technique présentée ici a été développée pour la recherche et n'est pas pour l'heure commercialisable ; elle est cependant opérationnelle. Nous l'utilisons couramment pour décrire à l'échelle métrique des tronçons de 2 à $3 \mathrm{~km}$ de cours d'eau. Son domaine d'application possible est vaste : 
- la constitution de jeux de données pour la mise au point de modèles hydrauliques, ligne d'eau, champs de vitesses, transport solide. La chaîne de traitement se limite pour l'instant au débit topographié ; nous souhaitons, à l'avenir, intégrer un modèle hydraulique d'évolution de la ligne d'eau en fonction du débit,

- la géomorphologie fluviale, pour la comparaison d'états de rivière avant et après une crue par exemple,

- l'étude du fonctionnement biologique par la caractérisation des relations habitats/invertébrés/poissons établies au travers des densités de colonisation et des flux d'organismes dérivants.

L'un des aspects les plus prometteurs de cette démarche réside dans les possibilités d'élaboration et de test des concepts d'ambiance qui déterminent les relations des espèces avec leurs habitats. En effet, le système offre une grande liberté de définition en terme de proximité de structures et d'agrégations pondérées de paramètres hydrauliques et morphologiques.

\section{BIBLIOGRAPHIE}

BOVEE K.D., 1981. A user guide to the Instream Flow Incremental Methodology. U.S. Fish and Wildlife Service. Biological Services Program, Rep. $n^{\circ}$ FWS/0BS 80/52, $232 \mathrm{p}$.

BOVEE K.D., 1982. A guide to stream habitat analysis using the Instream Flow Incremental Methodology. Instream flow information paper $n^{\circ} 12$, Cooperative Instream Flow Service Group, Fish and Wildlife Service, U.S. Department of the Interior, FWS/OBS $82 / 26,248 \mathrm{p}$.

CARREL G., DUMONT B., LE COARER Y., RIVIER B., 1994. Morphodynamique et biologie piscicole de la Durance dans le secteur d'Espinasses - Rochebrune. Rapport CEMAGREF Aix-en-Provence, $45 \mathrm{p}$.

LECLERC M., BOUdREAU P., BECHARA J., BELZILE L., VILLENEUVE D., 1994. Modélisation de la dynamique de l'habitat des jeunes stades de saumon atlantique (Salmo salar) de la rivière Ashuapmushuan (Québec, Canada). Bull. Fr. Pêche Piscic., 332, 11-32.

LIMERINOS J.T., 1970. Determination of the Manning coefficient from measured bed roughness in natural channels. U.S. Geol. Survey Water Supply paper, 1898 B, 47 p.

PARUSSIE M., 1994. Modélisations des relations habitat/truite fario dans le cas d'une rivière à débit réservé : la Durance à l'aval de Serre-Ponçon. Mémoire de 3ème année, Ecole Nationale du Génie de l'Eau et de l'Environnement de Strasbourg, $100 \mathrm{p}$.

SOUCHON Y., TROCHERIE F., FRAGNOUD E., LACOMBE C., 1989. Les modèles numériques des microhabitats des poissons : application et nouveaux développements. Sci. Eau, 2, 807-830. 